\title{
Guest Editorial: Retrospective cohort studies using the registry database of hematopoietic stem cell transplantation in Japan. How can we produce reliable Japanese evidences?
}

\author{
Yoshinobu Kanda ${ }^{1,2}$
}

Received: 7 October 2015 / Revised: 19 October 2015 / Accepted: 20 October 2015 / Published online: 28 October 2015

(c) The Japanese Society of Hematology 2015

Hematopoietic stem cell transplantation (HSCT) is an effective option for the treatment of hematological diseases; however, it is also associated with significant complications and treatment related deaths, especially after allogeneic HSCT. The conduct of clinical studies thus remains important in improving HSCT outcomes and establishing an indication for HSCT in the treatment of a variety of hematological diseases. Randomized controlled trials (RCT) produce evidence of the highest level, but it is impossible to perform RCT for every clinical question raised in daily practice. In addition, in Japan, the number of HSCT performed at individual transplantation centers is limited [1]. A large database of HSCT recipients is thus needed to enable retrospective cohort studies designed to produce meaningful evidence in the area of HSCT.

Fortunately, the Japan Society for Hematopoietic Cell Transplantation (JSHCT) began to collect data of HSCT performed in Japan more than two decades ago. The registry is currently managed by the Japanese Data Center for Hematopoietic Cell Transplantation and now includes data on approximately 80,000 HSCT cases [1]. The establishment of the JSHCT Working Groups in 2010 also accelerated the usage of the database. To date, more than 50 articles have been published through the activities of JSHCT Working Groups [2]. This body of evidence is especially important for Japanese patients and physicians, since the outcome of HSCT often differs between Japanese and Western patients.

Yoshinobu Kanda

ycanda-tky@umin.ac.jp

1 Division of Hematology, Jichi Medical University, 3311-1, Yakushiji, Shimotuke-city, Tochigi 329-0498, Japan

2 Division of Hematology, Saitama Medical Center, Jichi Medical University, Saitama, Japan
However, several concerns about the system remain. First, at most transplantation centers, data submission is performed by busy physicians, and it is not always simple for them to input HSCT data accurately. The current database, called Transplant Registry Unified management Program 2 (TRUMP2), incorporates a logical check program to help prevent missing data or inconsistencies among submitted data, but the inconsistencies between submitted data and patient clinical records cannot be detected. On-site auditing, as performed in the Center for International Blood and Marrow Transplant Research, is the best way to assure the quality of the database, but requires a huge investment of resources.

Second, retrospective cohort studies can be associated with significant biases. For example, in comparing two treatment strategies, the selection of a strategy is always affected by background factors specific to the patients, which may confound comparison. Statistical techniques to reduce the effect of biases are thus needed to establish more reliable conclusions. Japan, however, suffers from a shortage of biostatisticians to assist physicians in statistical analyses. In addition, the TRUMP2 database includes more than 1200 variables, the definitions of which are very complex. To address this problem, "shared scripts", that produce variables of unified definition for statistical analyses, have been investigated and distributed on the JSHCT homepage.

In the Progress in Hematology series in this issue of the International Journal of Hematology, three experts in this area review the current status and future direction of TRUMP2 registry data studies. I believe that these reviews will be of interest to the readers of the International Journal of Hematology, not restricted to transplantation physicians, as large database analyses are also important in areas outside HSCT studies. 


\section{References}

1. Hematopoietic Cell Transplantation in Japan annual report of Nationwide Survey 2014. http://www.jdchct.or.jp/data/ report/2014/2-2-1.pdf.
2. List of publications by the Working Groups of the Japan Society for Hematopoietic Cell Transplantation. http://www.jdchct.or.jp/ data/report/2014/4-2.pdf. 